\title{
RADON-NIKODYM DENSITIES BETWEEN HARMONIC MEASURES ON THE IDEAL BOUNDARY OF AN OPEN RIEMANN SURFACE
}

\author{
MITSURU NAKAI
}

Dedicated to the memory of Professor TAdasi Nakayama

1. Resolutive compactification and harmonic measures. Let $R$ be an open Riemann surface. A compact Hausdorff space $R^{*}$ containing $R$ as its dense subspace is called a compactification of $R$ and the compact set $\Delta=R^{*}-R$ is called an ideal boundary of $R$. Hereafter we always assume that $R$ does not belong to the class $O_{G}$. Given a real-valued function $f$ on $\Delta$, we denote by $\bar{\varphi}_{f}^{R, R^{*}}$ (resp. $\underline{\varphi}_{f}^{R, R^{*}}$ ) the totality of lower bounded superharmonic (resp. upper bounded subharmonic) functions $s$ on $R$ satisfying

$$
\lim \inf _{R \ni p \rightarrow p^{*}} s(p) \geq f\left(p^{*}\right) \quad\left(\text { resp. lim } \sup _{R \ni p \rightarrow p^{*}} s(p) \leq f\left(p^{*}\right)\right)
$$

for any point $p^{*}$ in $\Delta$. If these two families are not empty, then

$$
\bar{H}_{f}^{R, R^{*}}(p)=\inf \left(s(p) ; s \in \bar{\varphi}_{f}^{R, R^{*}}\right) \text { and } \underline{H}_{f}^{R, R^{*}}(p)=\sup \left(s(p) ; s \in \underline{\varphi} \underline{f}_{f}^{R, R^{*}}\right)
$$

are harmonic functions on $R$ and $\bar{H}_{f}^{R, R^{*}} \geq \underline{H}_{f}^{R, R^{*}}$ on $R$. If these two functions coincide with each other on $R$, then we denote by $H_{f}^{R, R^{*}}$ this common function and call $f$ resolutive with respect to $R^{*}$ (or $\Delta$ ). We denote by $C(\Delta)$ the totality of bounded real valued continuous functions on $\Delta$. If any function in $C(\Delta)$ is resolutive with respect to $\Delta$, then following Constantinescu and Cornea [1] we say that $R^{*}$ is a resolutive compactification of $R$. Important examples of resolutive compactifications are Wiener's, Martin's Royden's, Kuramochi's and Kerékjártó-Stoilow's compactifications (see [1]). Hereafter we always consider the resolutive compactification $R^{*}$ of $R$.

Fix a point $p$ in $R$. It is easy to see that $f \rightarrow H_{f}^{R, R^{*}}(p)$ is a positive linear functional on $C(\Delta)$ and so by Riesz-Markoff-Kakutani's theorem, there exists a positive regular Borel measure $\mu_{p}$ on $\Delta$ such that

Received February 24, 1965. 


$$
H_{f}^{R, R^{*}}(p)=\int_{\Delta} f\left(p^{*}\right) d_{\mu p}\left(p^{*}\right)
$$

The measure $\mu_{p}$ is called the harmonic measure on 1 with the reference point $p$. We shall investigate the interdependence between each members of the family $\left(\mu_{p} ; p \in R\right)$ of harmonic measures.

2. Harnack's function. Let $k$ be the Harnack's function on $R \times R$, i.e. the function $k$ defined by

$$
k\left(p, p^{\prime}\right)=\inf \left(c>0 ; c^{-1} u(p) \leq u\left(p^{\prime}\right) \leq c u(p) \text { for any } u \in H P(R)\right) .
$$

Then $1 \leq k\left(p, p^{\prime}\right)<\infty$ for any $p$ and $p^{\prime}$ in $R$ and $\lim _{p \rightarrow p^{\prime}} k\left(p, p^{\prime}\right)=1$. In fact, let $U$ be a relatively compact simply connected domain in $R$ containing $p$ and $p^{\prime}$, and $\phi$ a $1: 1$ conformal mapping of $U$ onto $(z ;|z|<1)$ with $\phi\left(p^{\prime}\right)=0$. Then by putting $\phi(p)=r e^{i t}$

$$
u(p)=(1 / 2 \pi) \int_{0}^{2 \pi}\left(\left(1-r^{2}\right) /\left(1-2 r \cos (\theta-t)+r^{2}\right)\right) u\left(\phi^{-1}\left(e^{i \theta}\right)\right) d \theta
$$

for any $u$ in $H P(R)$ and so

$$
((1-r) /(1+r)) u(p) \leq u\left(p^{\prime}\right) \leq((1+r) /(1-r)) u(p) .
$$

Thus $1 \leq k\left(p, p^{\prime}\right) \leq(1+r) /(1-r)<\infty$ and if $p \rightarrow p^{\prime}$, then $r \rightarrow 0$ and so $\lim _{p \rightarrow p} k\left(p, p^{\prime}\right)=1$. Moreover it is easy to see that $k(p, p)=1, k\left(p, p^{\prime}\right)=$ $k\left(p^{\prime}, p\right)$ and $k\left(p, p^{\prime \prime}\right) \leq k\left(p, p^{\prime}\right) k\left(p^{\prime}, p^{\prime \prime}\right)$ for any $p, p^{\prime}$ and $p^{\prime \prime}$ in $R$.

3. Harmonic kernel. Let $p$ and $q$ belong to $R$. By the definition of $k(p, q)$, we see that

$$
k(p, q)^{-1} d_{\mu_{q}} \leq d \mu_{p} \leq k(p, q) d \mu_{q} .
$$

Thus measures $\mu_{p}(p \in R)$ are absolutely continuous with respect to each other and so the $\mu_{p}$-integrability and the $\mu_{p}$-nullity do not depend on the special choice of $p$ in $R$. We denote by $\left(d \mu_{q} / d \mu_{p}\right)\left(p^{*}\right)$ the Radon-Nikodym density of $\mu_{q}$ with respect to $\mu_{p}$.

We fix a point $o$ in $R$. Then we can easily see that the function $p \rightarrow$ $\int_{\Delta} f\left(p^{*}\right) d \mu_{p}\left(p^{*}\right)$ is harmonic on $R$ if $f$ is $\mu_{o}$-integrable on $\Delta$. The main assertion in this note is the following

Theorem. There exists a function $P_{o}\left(p, p^{*}\right)$ on $R \times \Delta$ such that

(a) $P_{o}\left(p, p^{*}\right)=\left(d \mu_{p} / d_{\mu_{0}}\right)\left(p^{*}\right)$ ( $\mu_{0}$-almost everywhere $)$ on $\Delta$ as the function 
of $p^{*}$ for any fixed $p$ in $R$;

(b) $P_{o}\left(p, p^{*}\right)$ is harmonic on $R$ as the function of $p$ for any fixed $p^{*}$ in $\Delta$;

(c) $\dot{k}(o, p)^{-1} \leq P_{o}\left(p, p^{*}\right) \leq k(o, p)$ for any $\left(p, p^{*}\right)$ in $R \times \Delta$;

(d) $P_{o}\left(p, p^{*}\right)$ is Borel measurable on $R \times \Delta$ as the function of $\left(p, p^{*}\right)$.

Needless to say, such a function $P_{o}\left(p, p^{*}\right)$ is not unique in the proper sense, but unique in the following sense: if $\widetilde{P}_{o}\left(p, p^{*}\right)$ is another function on $R \times \Delta$ satisfying the above four conditions, then $P_{o}\left(p, p^{*}\right)=\widetilde{P}_{c}\left(p, p^{*}\right) \widetilde{\mu}_{o}$-almost everywhere on $R \times 4$. Here $\widetilde{\mu}_{o}$ is the product measure $\widetilde{\mu} \times \mu_{0}$, where $\widetilde{\mu}$ is a measure on $R$ which is equivalent to the Lebesgue measure in each parameter neighborhood of $R$. Such a $P_{o}\left(p, p^{*}\right)$ may be called a harmonic kernel (or Poisson type kernel) on $R \times \Delta$ with the reference point $o$. For any Borel function $f, \mu_{o}$-integrable on 4 , we then have

$$
H_{f}^{R, R^{*}}(p)=\int_{\Delta} P_{o}\left(p, p^{*}\right) f\left(p^{*}\right) d \mu_{o}\left(p^{*}\right)
$$

The harmonicity of the function $p \rightarrow P_{o}\left(p, p^{*}\right)$ increases the usefulness of the above integral representation.

4. Proof of Theorem. Let $\widetilde{P}\left(p, p^{*}\right)$ be an arbitrary but fixed function on $R \times \Delta$ such that $\widetilde{P}\left(p, p^{*}\right)=\left(d \mu_{p} / d \mu_{o}\right)\left(p^{*}\right)\left(\mu_{o}\right.$-almost everywhere $)$ on $\Delta$ as the function of $p^{*}$ for any fixed $p$ in $R$. We may assume that $\widetilde{P}\left(o, p^{*}\right) \equiv 1$ on $\Delta$. Since $R$ is separable, there exists a countable dense subset $D$ of $R$ with $o \in D$.

For any $p$ and $q$ in $D$, by (1), we see that

$$
k(p, q)^{-1}\left(d \mu_{q} / d \mu_{o}\right)\left(p^{*}\right) \leq\left(d \mu_{p} / d \mu_{o}\right)\left(p^{*}\right) \leq k(p, q)\left(d \mu_{q} / d \mu_{o}\right)\left(p^{*}\right)
$$

$\mu_{0}$-almost everywhere on $\Delta$ as the function of $p^{*}$. Hence there exists a Borel set $E(p, q)$ in $\Delta$ such that

and

$$
\mu_{o}(E(p, q))=0
$$

$$
k(p, q)^{-1} \widetilde{P}\left(q, p^{*}\right) \leq \widetilde{P}\left(p, p^{*}\right) \leq k(p, q) \widetilde{P}\left(q, p^{*}\right)
$$

for any $p^{*}$ in $\Delta-E(p, q)$. Let

$$
E=\cup_{p, q \in D} E(p, q) .
$$

Since $D$ is countable, $\mu_{o}(E)=0$. Hence

$$
k(p, q)^{-1} \widetilde{P}\left(q, p^{*}\right) \leq \widetilde{P}\left(p, p^{*}\right) \leq k(p, q) \widetilde{P}\left(q, p^{*}\right)
$$


for any $p$ and $q$ in $D$ and $p^{*}$ in $\Delta-E$. In particular, since $\widetilde{P}\left(o, p^{*}\right)=1$ on $\Delta$,

$$
k(p, o)^{-1} \leq \widetilde{P}\left(p, p^{*}\right) \leq k(p, o)
$$

for any $p$ in $D$ and $p^{*}$ in $\Delta-E$. Thus

$$
\left|\widetilde{P}\left(p, p^{*}\right)-\widetilde{P}\left(q, p^{*}\right)\right| \leq k(p, o) \max \left(k(p, q)-1,1-k(p, q)^{-1}\right)
$$

for any $p$ and $q$ in $D$ and $p^{*}$ in $\Delta-E$. We saw in Section 2 that

$$
1 \leq k(p, q) \leq k\left(p, p_{0}\right) k\left(p, q_{0}\right), \quad 1 \leq k(p, o) \leq k\left(o, p_{0}\right) k\left(p, p_{0}\right)
$$

and

$$
\lim _{D \ni p \rightarrow p_{0}} k\left(p, p_{0}\right)=\lim _{D \ni q \rightarrow p_{0}} k\left(q, p_{0}\right)=1
$$

for any $p_{0}$ in $R$. From these and (3), it follows that

$$
\lim _{D \ni p, q \rightarrow p_{0}}\left|\widetilde{P}\left(p, p^{*}\right)-\widetilde{P}\left(q, p^{*}\right)\right|=0,
$$

or equivalently that

$$
\lim _{D \ni p \rightarrow p_{0}} \widetilde{P}\left(p, p^{*}\right)
$$

exists for any $p_{0}$ in $R$ and if $p_{0}$ belongs to $D$, then

$$
\lim _{D \ni p \rightarrow p_{0}} \widetilde{P}\left(p, p^{*}\right)=\widetilde{P}\left(p_{0}, p^{*}\right) .
$$

Hence if we set

$$
P\left(p, p^{*}\right)=\lim _{D \ni p^{\prime} \rightarrow p} \tilde{P}\left(p^{\prime}, p^{*}\right)
$$

in $R \times(\Delta-E)$, then the function $p \rightarrow P\left(p, p^{*}\right)$ is continuous on $R$ for fixed $p^{*}$ in $\Delta-E$. For arbitrary point $p$ in $R$, take a sequence $\left(p_{n}\right)_{n=1}^{\infty}$ of points in $D$ with $p_{n} \rightarrow p$. Then for any function $f$ in $C(\Delta)$, by using (2), the definition of $P\left(p, p^{*}\right)$ and Lebesgue's convergence theorem,

$$
\begin{aligned}
\int_{\Delta} f\left(p^{*}\right) d \mu_{p}\left(p^{*}\right) & =H_{f}^{R, R^{*}}(p) \\
& =\lim _{n \rightarrow \infty} H_{f}^{R, R^{*}}\left(p_{n}\right) \\
& =\lim _{n \rightarrow \infty} \int_{\Delta-E} \widetilde{P}\left(p_{n}, p^{*}\right) f\left(p^{*}\right) d \mu_{o}\left(p^{*}\right) \\
& =\int_{\Delta-E} \lim _{n \rightarrow \infty} \widetilde{P}\left(p_{n}, p^{*}\right) f\left(p^{*}\right) d \mu_{o}\left(p^{*}\right) \\
& =\int_{\Delta-E} P\left(p, p^{*}\right) f\left(p^{*}\right) d \mu_{o}\left(p^{*}\right) .
\end{aligned}
$$

This shows that $d \mu_{p}\left(p^{*}\right)=P\left(p, p^{*}\right) d \mu_{o}\left(p^{*}\right)$. Hence $P\left(p, p^{*}\right)=\left(d \mu_{p} / d \mu_{0}\right)\left(p^{*}\right)$ 
$\mu_{0}$-almost everwhere.

Let $\phi$ be an analytic mapping of the open unit disc $(z ;|z|<1)$ onto $R$. Now we prove that the function $p \rightarrow P\left(p, p^{*}\right)$ is harmonic on $R$ for almost every fixed $p^{*}$ in $\Delta$. For the aim, we have only to show that the function $z \rightarrow P\left(\phi(z), p^{*}\right)$ is harmonic on $(z ;|z|<1)$ for almost every fixed $p^{*}$ in $\Delta$, since $p \rightarrow P\left(p, p^{*}\right)$ is continuous on $R$ for any fixed $p^{*}$ in $\Delta-E$. Since $p^{*} \rightarrow P\left(\phi(z), p^{*}\right)$ is Borel measurable on $\Delta$ for any fixed $z$ in $(z ;|z|<1)$ and $z \rightarrow P\left(\phi(z), p^{*}\right)$ is continuous on $R$ for any fixed $p^{*}$ in $\Delta-E$, it is easy to see that the function $\left(z, p^{*}\right) \rightarrow P\left(\phi(z), p^{*}\right)$ is Borel measurable on $R \times \Delta$.

Let $\left(z_{n}\right)_{n=1}^{\infty}$ be a countable dense subset of $(z ;|z|<1)$. Fix an arbitrary positive integer $n$ and choose a countable dense subset $\left(r_{m}\right)_{m=1}^{\infty}$ of the open interval $\left(0,1-\left|z_{n}\right|\right)$. Then for any $f$ in $C(\Delta)$, since $\int_{\Delta} P\left(\phi(z), p^{*} \mid f\left(p^{*}\right) d \mu_{o}\left(p^{*}\right)\right.$ is harmonic in $z$ of $(z ;|z|<1)$, by Fubini's theorem,

$$
\begin{aligned}
\int_{\Delta} P\left(\phi\left(z_{n}\right), p^{*}\right) f\left(p^{*}\right) d \mu_{0}\left(p^{*}\right) & =\frac{1}{2 \pi} \int_{0}^{2 \pi}\left[\int_{\Delta} P\left(\phi\left(z_{n}+r_{m} e^{i \theta}\right), p^{*}\right) f\left(p^{*}\right) d \mu_{o}\left(p^{*}\right)\right] d \theta \\
& =\int_{\Delta}\left[\frac{1}{2 \pi} \int_{0}^{2 \pi} P\left(\phi\left(z_{n}+r_{m} e^{i \theta}\right), p^{*}\right) d \theta\right] f\left(p^{*}\right) d \mu_{o}\left(p^{*}\right)
\end{aligned}
$$

Hence there exists a set $F_{n, m}$ in $\Delta$ with $\mu_{o}\left(F_{n, m}\right)=0$ such that for any $p^{*}$ in $\Delta-F_{n, m}$ it holds that

$$
P\left(\phi\left(z_{n}\right), p^{*}\right)=\frac{1}{2 \pi} \int_{0}^{2 \pi} P\left(\phi\left(z_{n}+r_{m} e^{i \theta}\right), p^{*}\right) d \theta .
$$

Let $F_{n}=E \cup\left(\cup{ }_{m=1}^{\infty} F_{n, m}\right)$. Then $\mu_{o}\left(F_{n}\right)=0$ and the identity (4) holds for any $m=1,2, \ldots$ and $p^{*}$ in $\Delta-F_{n}$. By the continuity of $P\left(\phi(z), p^{*}\right)$ in $z$ for any fixed $p^{*}$ in $\Delta-E$, we conclude that

$$
P\left(\phi\left(z_{n}\right), p^{*}\right)=\frac{1}{2 \pi} \int_{0}^{2 \pi} P\left(\phi\left(z_{n}+r e^{i \theta}\right), p^{*}\right) d \theta
$$

for any $r$ in $0<r<1-\left|z_{n}\right|$ and $p^{*}$ in $\Delta-F_{n}$. Finally let $F=\cup_{n=1}^{\infty} F_{n}$. Then $\mu_{o}(F)=0$ and (5) holds for any $n=1,2, \ldots$ and any $r$ in $0<r<1-\left|z_{n}\right|$ and any $p^{*}$ in $\Delta-F$. By the continuity of $P\left(\phi(z), p^{*}\right)$ in $z$ for any fixed $p^{*}$ in $\Delta-E$, we conclude that

$$
P\left(\phi(z), p^{*}\right)=\frac{1}{2 \pi} \int_{0}^{2 \pi} P\left(\phi\left(z+r e^{i \theta}\right), p^{*}\right) d \theta
$$

for any $z$ in the unit disc and $r$ in $0<r<1-|z|$ and $p^{*}$ in $A-F$, which shows 
that $z \rightarrow P\left(\phi(z), p^{*}\right)$ is harmonic in $(z ;|z|<1)$ for any fixed $p^{*}$ in $\Delta-F$.

Thus the function $p \rightarrow P\left(p, p^{*}\right)$ is harmonic on $R$ for any fixed $p^{*}$ in $\Delta-F$ with $\mu_{0}(F)=0$. Let

$$
P_{o}\left(p, p^{*}\right)= \begin{cases}P\left(p, p^{*}\right), & \text { for }\left(p, p^{*}\right) \text { in } R \times(\Delta-F) ; \\ 1, & \text { for }\left(p, p^{*}\right) \text { in } R \times F .\end{cases}
$$

Then for any fixed $p$ in $R, P_{o}\left(p, p^{*}\right)=P\left(p, p^{*}\right)=\left(d \mu_{p} / d \mu_{0}\right)\left(p^{*}\right)\left(\mu_{o}\right.$-almost everywhere) on $\Delta$. Thus $(a)$ is satisfied by $P_{o}\left(p, p^{*}\right)$ thus constructed. It is also clear that $P_{o}\left(p, p^{*}\right)$ satisfies $(b)$. The condition $(c)$ follows immediately from $(b)$, the definition of $k(o, p)$ and the fact that $P_{o}\left(o, p^{*}\right) \equiv 1$ for any $p^{*}$ in $\Delta$ (see (2)). The last condition $(d)$ is an easy consequence of $(a)$ and $(b)$.

\section{REFERENCES}

[1] C. Constantinescu-A. Cornea: Ideale Ränder Riemannscher Flächen, Springer-Verlag, 1963.

Mathematical Institute

Nagoya University 